\title{
A New Face Recognition Method Based On SVD
}

\author{
Yuanyuan Ma, Dengyin Zhang
}

Institute of communication and Information Engineering, Nanjing University of Posts and Telecommunications, Nanjing

210003, China

Key words: face recognition; singular value decomposition; singular value vector; matrix similarity

\begin{abstract}
In order to further improve the accuracy of face recognition and reduce the time complexity, this paper presents a new method of face recognition based on the singular value decomposition (SVD). Combining the characteristics of singular value vector (SVV) and orthogonal matrix similarity, a new criterion is established to obtain the decision category of the face to be tested. The ORL face database is used to verify the recognition effect of the proposed method. Experiment results show that the proposed method is superior to other methods in recognition rate and time complexity.
\end{abstract}

\section{Introduction}

Face recognition is a biometric identification method with high recognition accuracy and low invasiveness. In the early 1970s, face recognition has attracted wide attention of experts from various fields, such as computer vision, graphics and so on. Many achievements have been made in the field of face recognition [1], but there are still two issues that how to extract proper recognition features and design corresponding classifiers. SVD is an effective method to extract image features, which not only has good stability for transposition, translation, rotation and image transformation, but also has good robustness to noise and light intensity transformation [2]. Therefore, the SVV is used as a distinguishing characteristic in a large number of algorithms. Hong first proposed the use of facial image singular value as a recognition feature [3], but the algorithm's accurate recognition rate was only $57.33 \%$. Hong believed that a small sample problem made the recognition rate too low.
Tian and others found out that most of the information of the human face is contained in the orthogonal matrices obtained by SVD, while the singular value itself has less effective face information [4]. In the literature [5], a fusion of local and global singular value method was proposed for identification, which improved the recognition rate to some extent, but the information contained in left and right orthogonal matrices was still not effectively utilized and the algorithm was complex and time-consuming. The concept of matrix similarity was first proposed and applied to image feature extraction in the literature [6], achieving a good result. Based on the above research, a new method of face recognition based on SVD is proposed, which integrates the concept of matrix similarity into SVD. A criterion fusing SVV feature and matrix similarity feature is put forward to make the best use of effective information obtained by SVD. Experiments are performed on ORL face database. Comparing the proposed method with other four SVD-based face recognition methods, the results show that the proposed method is superior to other methods in recognition rate and time complexity.

\section{SVD principle}

SVD is a very effective processing tool for image content analysis. It can decompose any kind of array into a lower-dimensional matrix to capture image structure information. We can start with the concept of singular value and SVD to find the appropriate information to describe human face.

If there is a matrix $A \in R^{m \times n}$, then there exist orthogonal matrices $U=\left[u_{1}, u_{2}, \ldots, u_{m}\right] \in R^{m \times m}, V=\left[v_{1}, v_{2}, \ldots, v_{n}\right] \in R^{n \times n}$, variable $P=\min (m, n)$, making

$$
U^{T} A V=\operatorname{diag}\left[\sigma_{1}, \sigma_{2}, \ldots, \sigma_{P}\right]=W
$$




$$
A=U W V^{T}
$$

Where $\sigma_{1} \geq \sigma_{2} \geq \ldots \geq \sigma_{\mathrm{p}} \geq 0, \sigma_{i}(i=1,2, \ldots, P)$ is called the singular value of $A$, and it is also the square root of $\lambda_{i}$ which is the eigenvalue of $A A^{\mathrm{T}}$ or $A^{\mathrm{T}} A . u_{i}(i=1,2, \ldots, m)$ is a unit orthogonal eigenvector of $A A^{\mathrm{T}}$, called the left singular vector of $A ; v_{i}(i=1,2, \ldots, n)$ is a unit orthogonal eigenvector of $A^{\mathrm{T}} A$, called the right singular vector of $A$.

The singular values arranged in descending order, $\left(\sigma_{1}\right.$, $\left.\sigma_{2}, \ldots, \sigma_{P}\right)$ is the SVV of $A$. Experiments in literature [7] show that a great deal of valid information of a image is included in the largest top $\mathrm{K}$ singular values and their corresponding left and right singular vectors, so we take $\left(\sigma_{1}\right.$, $\left.\sigma_{2}, \ldots, \sigma_{K}\right)$ as the SVV of the image.

In this paper, training sample images of the same kind of person need to be averaged as template image. Singular values of the template image are obtained using SVD. The vector $P_{B}=\left(\sigma_{B 1}, \sigma_{B 2}, \ldots, \sigma_{B K}\right)$ composed of the largest top $\mathrm{K}$ singular values is taken as the template SVV. Do the same operation to any testing sample image to get the testing SVV $P_{C}=\left(\sigma_{C 1}, \sigma_{C 2}, \ldots, \sigma_{C K}\right)$. Try to obtain Euclidean distance between the template SVV and the testing SVV.

$$
d_{B C}=\sqrt{\sum_{i=1}^{K}\left(\sigma_{B i}-\sigma_{C i}\right)^{2}}
$$

The smaller the Euclidean distance, the closer the relationship between the template SVV and the testing SVV is, then the more likely they belong to the same person.

\section{Matrix similarity}

In order to more effectively use the information of left and right orthogonal matrices obtained by SVD, the similarity degree of orthogonal matrices is introduced to help identify the face. Following are the concept of matrix similarity. Assuming $C^{m \times n}$ represents all $m \times n$ matrix, if there are $A, B$ $\in C^{m \times n}$, the inner product of the matrix is defined as below:

$$
\langle A, B\rangle=\operatorname{tr}\left(B^{T} A\right)
$$

$\operatorname{tr}(\cdot)$ represents the sum of the main diagonal elements of the matrix. Matrix inner product represents the sum of elements product of the corresponding positions of the two matrices. The norm of $\mathrm{A}$ is shown as below:

$$
\|A\|=\langle A, A\rangle^{1 / 2}
$$

The similarity of $A$ and $B$ is defined as below:

$$
\alpha=\cos \theta=\frac{\langle A, B\rangle}{\|A\| \bullet\|B\|}
$$

$\theta$ represents the angle between two matrices, whose range is $[-1,1]$. When $\theta=90^{\circ}$, then $\alpha=0$, illustrate there is no correlation between the two matrices; when $\theta=0^{\circ}$, then $\alpha=1$, illustrate there is the best correlation between the two matrices.

From the second chapter of this paper, we know that SVD produces two orthogonal matrices $U$ and $V$. Since a large amount of valid information is included in the largest top $\mathrm{K}$ singular values and their corresponding left and right singular vectors, we use the two orthogonal matrices $U_{B}$ and $V_{B}$ respectively composed of the left singular vector and the right singular vector corresponding to the top $\mathrm{K}$ singular values as the template orthogonal matrices. Do the same operation to the testing sample image, obtaining the testing orthogonal matrices $U_{C}$ and $V_{C}$. The discriminant of similarity between template orthogonal matrices and testing orthogonal matrices is as below:

$$
\beta_{B C}=\alpha_{U}+\alpha_{V}=\frac{\left\langle U_{B}, U_{C}\right\rangle}{\left\|U_{B}\right\| \bullet\left\|U_{C}\right\|}+\frac{\left\langle V_{B}, V_{C}\right\rangle}{\left\|V_{B}\right\| \bullet\left\|V_{C}\right\|}
$$

In which $\beta_{B C}$ is the similarity discrimination sign. The closer the value of $\beta_{B C}$ to 2 , the higher the similarity degree of orthogonal matrices is. Fusing matrix similarity discrimination and SVV identification, a new face recognition criterion is brought as below:

$$
\gamma=\frac{\beta_{B C}}{d_{B C}+\varepsilon}=\frac{\frac{\left\langle U_{B}, U_{C}\right\rangle}{\left\|U_{B}\right\| \bullet\left\|U_{C}\right\|}+\frac{\left\langle V_{B}, V_{C}\right\rangle}{\left\|V_{B}\right\| \bullet\left\|V_{C}\right\|}}{\sqrt{\sum_{i=1}^{K}\left(\sigma_{B i}-\sigma_{C i}\right)^{2}}+\varepsilon}
$$

In which $\varepsilon$ is a positive number added in the denominator to avoid the possibility of a denominator of zero. In practice, we take $\varepsilon=1 . \gamma$ is defined as the distinguishing flag of face recognition. According to the analysis of the previous, it can be drawn that the larger the value of $\gamma$, the closer the relationship between the testing sample image and the template image is. When the value of $\gamma$ is max, the character 
class to which the template image belongs is determined as the final recognition result.

\section{The basic steps of proposed method}

Suppose there are $\mathrm{N}$ human faces in the face database and each person has $M$ training sample images. If there is a testing sample image, the steps of determining the class of the image are as follows:

Step 1: The template image of the $i$ th $(i=1,2,3, \ldots, \mathrm{N})$ person is obtained by averaging his $M$ training sample images, then there are $\mathrm{N}$ template images in the database.

Step 2: SVD is performed for every template image to obtain the template $\operatorname{SVV} P_{B i}(i=1,2,3, \ldots, \mathrm{N})$ and the template orthogonal matrices $U_{B i}$ and $V_{B i}(i=1,2,3, \ldots, \mathrm{N})$.

Step 3: Similarly, SVD is performed for the testing sample image to obtain the testing SVV $P_{C}$ and the testing orthogonal matrix $U_{C}$ and $V_{C}$.

Step 4: According to formula (8), we can get the discrimination flag $\gamma_{i}(i=1,2,3, \ldots, \mathrm{N})$ between the testing sample image and each template image. We take the character category corresponding to the largest $\gamma_{i}$ as the category to which the testing sample image belongs.

\section{Experiment results and analysis}

The ORL face database is used to do experiments to analyze the effectiveness of the proposed method. The face database contains 400 face images, covering 40 people, each with 10 images of $112 \times 92$ pixels. These images are shot with different angles, different expressions, different light intensity, containing a wide variety of situations. Figure 1 shows ten images of a person in the face database.
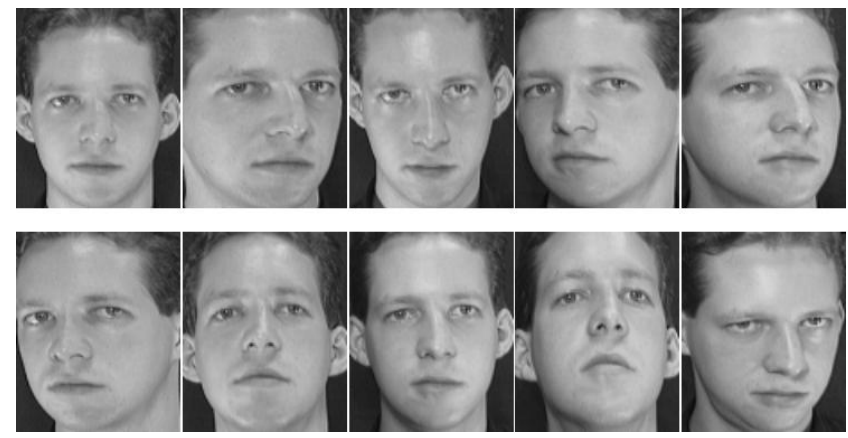

Fig.1: A person's 10 face images in ORL face database

In order to verify the effectiveness of the proposed method, two sets of experiments are conducted.

Exp.1: This experiment is to examine the influence of the variation of the SVV dimension $\mathrm{K}$ and the changing of training samples size $M$ on the recognition rate. In the ORL database, 1, 3, 5, 7 ( ie $M=1,3,5,7$ ) images of each person are respectively taken as the training samples, the remaining images as the samples to be tested, observing the change of recognition rate with $\mathrm{K}$ taking $1,2,3, \ldots, 20$. To ensure the accuracy of the experiment results, 800 experiments are carried out in all. 200 experiments are done when $M$ take any value in the $1,3,5,7$. When $M$ take a certain value, 10 experiments are performed no matter which value $\mathrm{K}$ takes in $1,2,3, . ., 20$, and average recognition rate of 10 experiments. The results are shown in Figure 2.

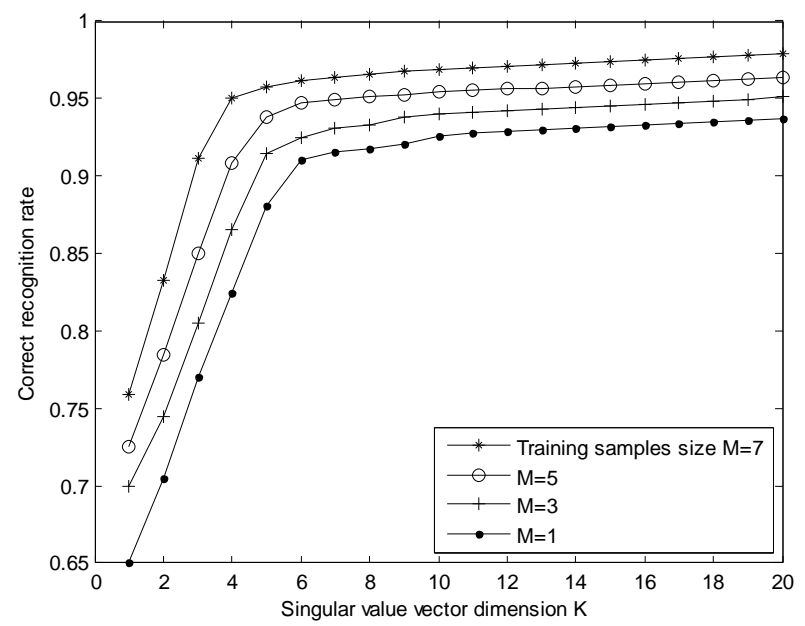

Fig.2: Experiment result

As can be seen from Figure 2, the recognition rate increases with the increasing of the dimension $\mathrm{K}$ and the size $\mathrm{M}$. In the initial stage of the change of dimension $K$, the recognition rate has a very significant increase; but when $\mathrm{K}$ grows to a certain stage, the growth of recognition rate obviously slows down and tends to a stable value. This is because the SVV consisted of the greatest top $\mathrm{K}$ singular value and the orthogonal matrices composed of the left and right singular vector corresponding to the top $\mathrm{K}$ singular value contain the main features of the image. The effect of training samples size $\mathrm{M}$ on the recognition rate is that when $\mathrm{M}$ is large, even if $\mathrm{K}$ is small, it can get a high recognition performance. For example, when $\mathrm{M}$ is $7, \mathrm{~K}$ is only 3 , it can achieve $91.5 \%$ recognition rate. It indicates that increasing the number of training samples is an effective way to 
improve the identification accuracy.

Exp.2: Compare the differences in recognition rate and time complexity between the proposed method and the other four SVD-based recognition methods. We characterize the time complexity in terms of time spent, including training time and recognition time. The 8 images of each person are randomly taken as the training sample images, the rest as testing images. $\mathrm{K}$ is taken as 4 . To reduce the experimental error and ensure the accuracy of the experimental results, we conduct 500 experiments and average the recognition rate and consumption time. The results are shown in Table 1.

Table 1: Performance comparison

\begin{tabular}{|l|c|c|}
\hline Methods & $\begin{array}{l}\text { Recognition } \\
\text { rate/\% }\end{array}$ & $\begin{array}{l}\text { Time } \\
\text { consumption/ms }\end{array}$ \\
\hline $\begin{array}{l}\text { Proposed } \\
\text { method }\end{array}$ & 96.7 & 285 \\
\hline $\begin{array}{l}\text { Literature[5] } \\
\text { method }\end{array}$ & 90.5 & 1128 \\
\hline $\begin{array}{l}\text { Literature[7] } \\
\text { method }\end{array}$ & 84.89 & 447 \\
\hline $\begin{array}{l}\text { Literature[8] } \\
\text { method }\end{array}$ & 93.8 & 561 \\
\hline $\begin{array}{l}\text { Literature[9] } \\
\text { method }\end{array}$ & 94.2 & 803 \\
\hline
\end{tabular}

It can be seen from Table 1 that the recognition rate of the proposed method is obviously superior to other conventional methods, and the time consumption in the whole process is significantly less than other methods. The reason behind this is that the training phase of obtaining the template features takes little time, and the calculation amount of the recognition phase is small.

\section{Conclusion}

In this paper, a new method of face recognition based on SVD is proposed to improve the recognition precision and reduce time complexity. The method uses the SVV feature and the orthogonal matrix similarity feature to establish new distinguishing criterion, making full use of the effective face information obtained by SVD. The proposed method has less time consumption and higher recognition accuracy than conventional methods.

\section{Acknowledgements}

The National Natural Science Foundation of China [61571241], Industry-university-research Prospective joint project of Jiangsu Province [BY2014014], and Major projects of Jiangsu Province university natural science research [15KJA510002].

\section{References}

[1]Tplba A S, El-Baz A H, El-Hary A A. "Face recognition: A literature review", Signal Processing, 2, pp. 88-103, (2005).

[2]Chen Liangyu, Zhu Zhenfu, Liu Zhongling, et al. "Analysis and application of image singular value feature vector scaling invariance", Infrared and Laser Engineering, 32, pp. 498-501, (2003).

[3]ZQ Hong. "Algebraic feature extraction of image for recognition", Pattern recognition, 24, pp. 211-219, (1992).

[4]Y Tian, T Tan, Y Wang, Y Fang. "Do singular values contain adequate information for face recognition?", Pattern recognition, 36, pp. 649-655, (2003)

[5] Zhang Cixiang, Liu Hui, Qiang Zhenping. "Face recognition based on sparse representation and singular value decomposition", Journal of Computer Applications, 33, pp. 233-235, (2013).

[6] Cheng Yongqing, Zhuang Yongming, Yang Jingyu. "Image feature extraction and recognition based on matrix similarity", Journal of Computer Research and Development, 11, pp. 42-48, (1992).

[7]Sun Jingjing, Zhang Hongfei, Sun Chang. "A new method of face recognition based on singular value decomposition", Science Technology and Engineering, 10, pp. 6204-6208, (2010).

[8]Wang Yunhong, Tan Tieniu, Zhu Yong. "Face Recognition Based on Singular Value Decomposition and Data Fusion", Chinese Journal of Computers, 23, pp. 649-653, (2000)

[9]He Jing, Feng Guocan. "The Application of Singular Value Decomposition in Face Recognition", Journal of Guangdong Education Institute, 26, pp. 92-96, (2005). 